\title{
On Harmonic Functions by using Ruscheweyh-Type Associated with Differential Operators
}

\author{
Mohammed A. Fathi \\ mohammed.csp120@student.uomosul.edu.iq \\ Department of Mathematics \\ Collage of Computer Science and Mathematics \\ University of Mosul
}

Received on: 29/11/2020

\author{
Abdul Rahman S. Juma \\ dr_juma@hotmail.com \\ College of Education for Pure \\ Science \\ University of Anbar
}

Accepted on: 10/01/2021

\begin{abstract}
By applying Ruscheweyh - type harmonic function on the class $\operatorname{AS}_{\mathrm{H}}(\lambda, \alpha, k, \gamma)$, a new subclass $\mathscr{H R}_{q}^{m}(m, \alpha, k, \gamma)$ for harmonic univalent function in the unit disk $\mathbb{D}$ is introduced, Furthermore, some geometric properties are obtained such as distortion theorem, sufficient coefficient bounds, extreme points and convex combination conditions for aforementioned subclass.
\end{abstract}

Keywords: Harmonic functions, Univalent function, Distortion theorem, Ruscheweyh type q- differential operator.

\section{Introduction.}

Let $\mathscr{H}$ be denoted to the class of all harmonic functions, consider $f=h+\bar{g}$ are univalent and orientation preserving in $U=\{z:|z|<1\}$.

$$
h(z)=z+\sum_{n=2}^{\infty} a_{n} z^{n} \text { and } g(z)=\sum_{n=1}^{\infty} b_{n} z^{n}, \quad\left|b_{1}\right|<1 \text {. }
$$

Normalized by $f(0)=0, f_{z}(0)=1$ where $f_{z}(0)$ denotes the derivative of $f(z)$ at $\mathrm{z}=0$ with $\mathrm{h}$ and $\mathrm{g}$ holomorphic part and co-holomorphic part of $f$ respectively, the necessary and sufficient condition make the function $f$ to be both locally univalent and orientation preserving in $\mathbb{D}$ is that $\left|h^{\prime}(z)\right|>\left|g^{\prime}(z)\right|$ in $\mathbb{D}$ (see [1]), we recall the notation of qdifference operator where Jackson[6] in 1909 initiated the application of q-calculus holomorphic univalent functions.

For $0<q<1$, Jackson's q-derivative of the $h(z)=z+\sum_{n=2}^{\infty} a_{n} z^{n}$, where $h$ is normalized holomorphic univalent functions.

$$
D_{q} h(z)=\left\{\begin{array}{cc}
\frac{h(z)-h(q z)}{(1-q) z}, & \text { for } z \neq 0 \\
h^{\prime}(0), & \text { for } z=0
\end{array}\right\},
$$

where $D_{q} h(z)=1+\sum_{n=2}^{\infty}[n]_{q} a_{n} z^{n-1}$ and $[n]_{q}=\frac{1-q^{n}}{1-q}$.

Kanas and Răducanu [5] introduced the Ruscheweyh type q- differential operator and investigated some properties for it. This operator has been studied by many researchers [2,7,8], Ruscheweyh type q-differential operator shown below.

$R_{q}^{m} h(z)=h(z) * F_{q, m+1}(z)=z+\sum_{n=2}^{\infty} \frac{\Gamma_{q}(n+m)}{(n-1) ! \Gamma_{q}(1+m)} a_{n} z^{n}, \quad m>-1$,

and have

$$
\begin{gathered}
F_{q, m+1}(z)=z+\sum_{n=2}^{\infty} \frac{\Gamma_{q}(n+m)}{(n-1) ! \Gamma_{q}(1+m)} z^{n} . \\
\text { Let } \emptyset(n, m)=\frac{\Gamma_{q}(n+m)}{(n-1) ! \Gamma_{q}(1+m)} .
\end{gathered}
$$

So $R_{q}^{m} h(z)=z+\sum_{n=2}^{\infty} \emptyset(n, m) a_{n} z^{n}, \quad m>-1$.

Also, 


$$
\begin{aligned}
& D_{q} R_{q}^{m}\left(h(z)=1+\sum_{n=2}^{\infty}[n]_{q} \emptyset(n, m) a_{n} z^{n-1} .\right. \\
& D_{q}\left(D_{q} R_{q}^{m}(h(z))=\sum_{n=2}^{\infty}[n]_{q}(n-1)_{q} \emptyset(n, m) a_{n} z^{n-2} .\right. \\
& D_{q} R_{q}^{m}(g(z))=\sum_{n=1}^{\infty}[n]_{q} \emptyset(n, m) b_{n} z^{n-1} . \\
& D_{q}\left(D_{q} \mathrm{R}_{\mathrm{q}}^{\mathrm{m}}(\mathrm{g}(\mathrm{z}))\right)=\sum_{\mathrm{n}=2}^{\infty}[\mathrm{n}]_{\mathrm{q}}[\mathrm{n}-1]_{\mathrm{q}} \emptyset(\mathrm{n}, \mathrm{m}) \mathrm{b}_{\mathrm{n}} \mathrm{z}^{\mathrm{n}-2}
\end{aligned}
$$

Juma and Kulkarni in [4] applied Ruscheweyh derivatives on the class $\operatorname{AS}_{\mathrm{H}}(\lambda, \alpha, k, \gamma)$. They obtained several geometric properties. We define a class of Ruscheweyh-type q-differential harmonic function $\mathscr{H} R_{q}^{m}(m, \alpha, k, \gamma)$ consisting of functions $f \in \mathcal{H}$ satisfying $f=h+\bar{g}$ where

$\mathrm{R}\left(\left(1+k e^{i \gamma}\right) \frac{z^{2} D_{q}{ }^{2}\left(R_{q}^{m} h(z)\right)+z D_{q}\left(R_{q}^{m} h(z)\right)+\overline{z^{2} D_{q}{ }^{2}\left(R_{q}^{m} g(z)\right)+z D_{q}\left(R_{q}^{m} g(z)\right)}}{z D_{q}\left(R_{q}^{m} h(z)\right)-\overline{z D_{q}\left(R_{q}^{m} h(z)\right)}}+1\right) \geq \alpha$,

where $0 \leq \alpha<1, \quad 0 \leq k<1, \lambda>-1$.

Let $\overline{\mathcal{H}}$ denoted to a subfamily of $\mathcal{H}$ consisting of the harmonic function, we define a subclass $\overline{\mathscr{H} R} R_{q}^{m}(m, \alpha, k, \gamma)=\mathscr{H} R_{q}^{m}(m, \alpha, k, \gamma) \cap \overline{\mathscr{H}}$ consisting harmonic function of the form.

$h(z)=z-\sum_{n=2}^{\infty} a_{n} z^{n}$ and $g(z)=\sum_{n=1}^{\infty} b_{n} z^{n}, a_{n} \geq 0, b_{n} \geq 0$.

\section{Main Result.}

Theorem 2.1. Let $f=h+\bar{g}$ with

$$
\begin{aligned}
\left(\sum_{n=1}^{\infty}[n]_{q} \varnothing(n,\right. & \left.m) \frac{\left[(1+k)[n-1]_{q}+2+k-\alpha\right]}{(4+2 k-\alpha)+(1+k)\left|b_{1}\right|}\right)\left|a_{n}\right| \\
& +\left(\sum_{n=1}^{\infty}[n]_{q} \varnothing(n, m) \frac{\left[(1+k)[n-1]_{q}+k-\alpha\right]}{(4+2 k-\alpha)+(1+k)\left|b_{1}\right|}\right)\left|b_{n}\right| \leq 1
\end{aligned}
$$

where $a_{1}=1,0 \leq \gamma<1,0 \leq \alpha<1,0 \leq k<1, m>-1$.Then $f \in \mathcal{H} R_{q}^{m}(m, \alpha, k, \gamma)$.

Proof. We will show that the coefficient estimate of the harmonic function $f=h+\bar{g}$ $\in \mathcal{H}$ satisfy inequality (2.1), therefore, $f=h+\bar{g}$ satisfies the condition (1.2),

$$
R\left(\left(1+k e^{i \gamma}\right) \frac{z^{2} D_{q}{ }^{2}\left(R_{q}^{m} h(z)\right)+z D_{q}\left(R_{q}^{m} h(z)\right)+\overline{z^{2} D_{q}{ }^{2}\left(R_{q}^{m} g(z)\right)+z D_{q}\left(R_{q}^{m} g(z)\right)}}{z D_{q}\left(R_{q}^{m} h(z)\right)-\overline{z D_{q}\left(R_{q}^{m} h(z)\right)}}+1\right) \geq \alpha
$$

Thus

$$
\begin{aligned}
& \mathrm{R}\left\{\frac{A(z)}{B(z)}\right\} \geq \alpha, \\
& \text { where } A(z)=\left(1+k e^{i \gamma}\right) z^{2} D_{q}{ }^{2}\left(R_{q}^{m} h(z)\right)+z D_{q}\left(R_{q}^{m} h(z)\right) \\
& +\overline{z^{2} D_{q}{ }^{2}\left(R_{q}^{m} g(z)\right)+z D_{q}\left(R_{q}^{m} g(z)\right)}+z D_{q}\left(R_{q}^{m} h(z)\right)-\overline{z D_{q}\left(R_{q}^{m} h(z)\right)} \text {. } \\
& A(z)=\left(2+k e^{i \gamma}\right) \mathrm{z}+k e^{i \gamma} \overline{b_{1}} \bar{z}+\sum_{\mathrm{n}=2}^{\infty}[\mathrm{n}]_{\mathrm{q}} \varnothing(\mathrm{n}, \mathrm{m})\left[\left(1+k e^{i \gamma}\right)[n-1]_{q}+2\right. \\
& \left.+k e^{i \gamma}\right] a_{n} z^{n}+\sum_{n=2}^{\infty}[n]_{q} \varnothing(\mathrm{n}, \mathrm{m})\left[\left(1+k e^{i \gamma}\right)[n-1]_{q}+k e^{i \gamma}\right] \overline{b_{n}} \bar{z}^{n} \text {. }
\end{aligned}
$$




$$
\begin{aligned}
B(z) & =z D_{q}\left(R_{q}^{m} h(z)\right)-\overline{z D_{q}\left(R_{q}^{m} h(z)\right)} \\
& =z-\overline{b_{1}} \bar{z}+\sum_{n=2}^{\infty}[n]_{q} \emptyset(n, m) a_{n} z^{n}-\sum_{n=2}^{\infty}[n]_{q} \emptyset(n, m) \overline{b_{n}} \bar{z}^{n} .
\end{aligned}
$$

Using the fact that $\mathrm{R}\{w\} \geq \alpha$ if and only if $|1-\alpha+w| \geq|1+\alpha-w|$, to prove that it is equivalent to show that

$$
\begin{aligned}
& |A(z)+(1-\alpha) B(z)|-|A(z)-(1+\alpha) B(z)| \geq \alpha . \\
& \text { We simplify the part }|A(z)+(1-\alpha) B(z)| \\
& =\mid\left(2+k e^{i \gamma}\right) z+k e^{i \gamma} \overline{b_{1}} \bar{z}+\sum_{n=2}^{\infty}[n]_{q} \emptyset(n, m)\left[\left(1+k e^{i \gamma}\right)[n-1]_{q}+2+k e^{i \gamma}\right] a_{n} z^{n} \\
& +\sum_{n=2}^{\infty}[n]_{q} \varnothing(n, m)\left[\left(1+k e^{i \gamma}\right)[n-1]_{q}+k e^{i \gamma}\right] \overline{b_{n}} \overline{z^{n}} \\
& +(1-\alpha)\left[z-\overline{b_{1}} \bar{z}+\sum_{n=2}^{\infty}[n]_{q} \varnothing(n, m) a_{n} z^{n}-\sum_{n=2}^{\infty}[n]_{q} \varnothing(n, m) \overline{b_{n}} \overline{z^{n}}\right] \mid- \\
& =\mid\left(2+k e^{i \gamma}+1-\alpha\right) z+\left(k e^{i \gamma} \overline{b_{1}}-\overline{b_{1}}+\alpha \overline{b_{1}}\right) \bar{z} \\
& +\sum_{n=2}^{\infty}[n]_{q} \varnothing(n, m)\left[\left(1+k e^{i \gamma}\right)[n-1]_{q}+2+k e^{i \gamma}+1-\alpha\right] a_{n} z^{n} \\
& +\sum_{n=2}^{\infty}[n]_{q} \varnothing(n, m)\left[\left(1+k e^{i \gamma}\right)[n-1]_{q}+k e^{i \gamma}-1+\alpha\right] \overline{b_{n}} \overline{z^{n}} \\
& \rightarrow|A(z)-(1-\alpha) B(z)|-|A(z)-(1+\alpha) B(z)| \\
& =\mid\left(3+k e^{i \gamma}-\alpha\right) z+\left(k e^{i \gamma}-1+\alpha\right) \overline{b_{1}} \bar{z} \\
& +\sum_{n=2}^{\infty}[n]_{q} \varnothing(n, m)\left[\left(1+k e^{i \gamma}\right)[n-1]_{q}+3+k e^{i \gamma}-\alpha\right] a_{n} z^{n} \\
& +\sum_{n=2}^{\infty}[n]_{q} \varnothing(n, m)\left[\left(1+k e^{i \gamma}\right)[n-1]_{q}+k e^{i \gamma}-1+\alpha\right] \overline{b_{n}} \overline{z^{n}} \\
& -\mid\left(1+k e^{i \gamma}-\alpha\right) z+\left(k e^{i \gamma}+1+\alpha\right) \overline{b_{1}} \bar{z} \\
& +\sum_{n=2}^{\infty}[n]_{q} \varnothing(n, m)\left[\left(1+k e^{i \gamma}\right)[n-1]_{q}+k e^{i \gamma}+1-\alpha\right] a_{n} z^{n} \\
& +\sum_{n=2}^{\infty}[n]_{q} \varnothing(n, m)\left[\left(1+k e^{i \gamma}\right)[n-1]_{q}+k e^{i \gamma}+1+\alpha\right] \overline{b_{n}} \overline{z^{n}} \mid \\
& \geq(3+k-\alpha)|z|-\left(k\left|b_{1}\right|-\left|b_{1}\right|+\alpha\left|b_{1}\right|\right)|z| \\
& -\sum_{n=2}^{\infty}[n]_{q} \varnothing(n, m)\left[(1+k)[n-1]_{q}+3+k-\alpha\right]\left|a_{n} \| z\right|^{n} \\
& -\sum_{n=2}^{\infty}[n]_{q} \varnothing(n, m)\left[(1+k)[n-1]_{q}+k-1+\alpha\right]\left|b_{n}\right||z|^{n} \\
& -\left((1+k-\alpha)|z|-\left(k\left|b_{1}\right|+\left|b_{1}\right|+\alpha\left|b_{1}\right|\right)|z|\right.
\end{aligned}
$$




$$
\begin{aligned}
& -\sum_{n=2}^{\infty}[n]_{q} \emptyset(n, m)\left[(1+k)[n-1]_{q}+k+1-\alpha\right]\left|a_{n}\right||z|^{n} \\
& \left.-\sum_{n=2}^{\infty}[n]_{q} \varnothing(n, m)\left[(1+k)[n-1]_{q}+k+1+\alpha\right]\left|b_{n}\right||z|^{n}\right) \\
& =2\left[(4+2 k-\alpha)+(1+k)\left|b_{1}\right|\right]|z| \\
& -\sum_{n=1}^{\infty}[n]_{q} \emptyset(n, m)\left[\left((1+k)[n-1]_{q}+2+k-\alpha\right)\right]\left|a_{n}\right||z|^{n} \\
& -\sum_{n=1}^{\infty}[n]_{q} \emptyset(n, m)\left[\left((1+k)[n-1]_{q}+k+\alpha\right)\right]\left|b_{n}\right||z|^{n} \\
& =2\left[(4+2 k-\alpha)+(1+k)\left|b_{1}\right|\right]|z|[1 \\
& -\sum_{n=1}^{\infty} \frac{[n]_{q} \phi(n, m)\left[\left((1+k)[n-1]_{q}+2+k-\alpha\right)\right]\left|a_{n}\right|}{\left[(4+2 k-\alpha)+(1+k)\left|b_{1}\right|\right]} \\
& \left.-\frac{\left.[n]_{q} \emptyset(n, m)\left[\left((1+k)[n-1]_{q}+k+\alpha\right)\right]\left|b_{n}\right|\right]}{\left[(4+2 k-\alpha)+(1+k)\left|b_{1}\right|\right]}\right]|z|^{n-1} .
\end{aligned}
$$

The expression shown above is non-negative from the inequality (2.1) and so $f \in \mathcal{H} \mathrm{R}_{\mathrm{q}}^{\mathrm{m}}(\mathrm{m}, \alpha, \mathrm{k}, \gamma)$.

Theorem 2.2. Let $f=h+\bar{g}$ given by

$$
\begin{aligned}
& f(z)=z-\sum_{n=2}^{\infty} a_{n} z^{n}+\sum_{n=1}^{\infty} \overline{b_{n} z^{n}} \in \overline{\mathcal{H}} R_{q}^{m}(\mathrm{~m}, \alpha, \mathrm{k}, \gamma) \text { if and only if : } \\
& \sum_{n=2}^{\infty} \frac{\left[\left((1+k)[n-1]_{q}+2+k-\alpha\right)\right]}{\left[(4+2 k-\alpha)(1+k)\left|b_{1}\right|\right]} a_{n}[n]_{q} \phi(n, m) \\
& +\sum_{n=1}^{\infty} \frac{[n]_{q} \emptyset(n, m)\left[\left((1+k)[n-1]_{q}+k+\alpha\right)\right] b_{n}}{\left[(4+2 k-\alpha)+(1+k)\left|b_{1}\right|\right]}<1,
\end{aligned}
$$

where $a_{1}=1$ and $0 \leq \alpha<1,0 \leq k \leq 1$

Proof. Since $\overline{\mathcal{H}} \mathrm{R}_{\mathrm{q}}^{\mathrm{m}}(\lambda, \alpha) \subset \mathcal{H} \mathrm{R}_{\mathrm{q}}^{\mathrm{m}}(\mathrm{m}, \alpha, \mathrm{k}, \gamma)$, if condition will satisfy, to prove the converse part. Start with function $f=h+\bar{g}$ in $\overline{\mathcal{H}} \mathrm{R}_{\mathrm{q}}^{\mathrm{m}}(\mathrm{m}, \alpha, \mathrm{k}, \gamma)$, we must have $\mathrm{R}\left(\left(1+k e^{i \gamma}\right) \frac{z^{2} D_{q}{ }^{2}\left(R_{q}^{m} h(z)\right)+z D_{q}\left(R_{q}^{m} h(z)\right)+\overline{z^{2} D_{q}{ }^{2}\left(R_{q}^{m} g(z)\right)+z D_{q}\left(R_{q}^{m} g(z)\right)}}{z D_{q}\left(R_{q}^{m} h(z)\right)-z D_{q}\left(R_{q}^{m} h(z)\right)}+1\right) \geq \alpha$ or equivalently:

$$
\begin{aligned}
& R\left(\frac{A(z)-\alpha B(z)}{B(z)}\right) \geq 0 \\
& R\left(\frac{\left((4+2 k-\alpha)(1+k)\left|b_{1}\right| z-\sum_{n=2}^{\infty}[n]_{q} \emptyset(n, m)\left[\left((1+k)[n-1]_{q}+2+k-\alpha\right)\right] a_{n} z^{n}\right)}{z-\overline{b_{1}} \bar{z}+\sum_{n=2}^{\infty}[n]_{q} \emptyset(n, m)[n]_{q} a_{n} z^{n}-\sum_{n=2}^{\infty}[n]_{q} \emptyset(n, m)[n]_{q} \overline{b_{n}} \overline{z^{n}}}\right)
\end{aligned}
$$

When the condition (2.3) not hold, the numerator in the above inequality will be negative for $\mathrm{r}$ goes to 1 . This condition for $f(z) \in \overline{\mathcal{H}} R_{q}^{m}(m, \alpha, k, \gamma)$. 
Recall that in [2] for a topological vector space $X$ over the field $\mathbb{C}$ of complex numbers, let $E \subseteq X$, the smallest convex set containing $E$ is called the closed convex hull of $E$ and denoted by $C l c o(E)$.

Theorem 2.3. A function $f(z) \in \overline{\mathcal{H}} R_{q}^{m}(\mathrm{~m}, \alpha, \mathrm{k}, \gamma)$ if and only if

$f(z)=\sum_{n=1}^{\infty}\left(M_{n} h_{n}(z)+S_{n} g_{n}(z)\right)$

where $h_{1}(z)=z_{1}$

$h_{n}(z)=z-\frac{\left[(4+2 k-\alpha)+(1+k)\left|b_{1}\right|\right]|z|}{\left[(1+k)[n-1]_{q}+2+k-\alpha\right] \varnothing(n, m)[n]_{q}} z^{n}$,

$n=2,3, \ldots$.

$g_{n}(z)=z-\frac{\left[(4+2 k-\alpha)+(1+k)\left|b_{1}\right|\right]|z|}{\left[(1+k)[n-1]_{q}+k-\alpha\right] \varnothing(n, m)[n]_{q}} \overline{z^{n}}$

$n=1,2,3, \ldots \ldots$

$\sum_{n=1}^{\infty}\left(M_{n}+S_{n}\right)=1, M_{n} \geq 0$ and $S_{n} \geq 0$.

In special case, the extreme point of $\overline{\mathcal{H}} R_{q}^{m}(\mathrm{~m}, \alpha, \mathrm{k}, \gamma)$ are $\left\{h_{n}\right\}$ and $\left\{g_{n}\right\}$.

Proof. Let $f$ be the function which can be written as (2.4), we have

$$
\begin{aligned}
& f(z)=\sum_{n=1}^{\infty}\left(M_{n}+S_{n}\right) z-\sum_{n=2}^{\infty} \frac{\left[(4+2 k-\alpha)+(1+k)\left|b_{1}\right|\right]}{\left[(1+k)[n-1]_{q}+2+k-\alpha\right] \varnothing(n, m)} M_{n} z^{n} \\
& -\sum_{n=1}^{\infty} \frac{\left[(4+2 k-\alpha)+(1+k)\left|b_{1}\right|\right]}{\left[(1+k)[n-1]_{q}+k-\alpha\right] \varnothing(n, m)} S_{n} \overline{z^{n}} \\
& =z-\sum_{n=2}^{\infty} a_{n} z^{n}-\sum_{n=1}^{\infty} b_{n} \overline{z^{n}}
\end{aligned}
$$

Therefore,

$$
\begin{aligned}
& \sum_{n=2}^{\infty}[n]_{q} \varnothing(n, m) \frac{\left[(1+k)[n-1]_{q}+k-\alpha\right]\left|a_{n}\right|}{\left[(4+2 k-\alpha)+(1+k)\left|b_{1}\right|\right]|z|} \\
& +\sum_{n=1}^{\infty}[n]_{q} \varnothing(n, m) \frac{\left[(1+k)[n-1]_{q}+k-\alpha\right]\left|b_{n}\right|}{\left[(4+2 k-\alpha)+(1+k)\left|b_{1}\right|\right]|z|} \\
& =\sum_{n=1}^{\infty} M_{n}+\sum_{n=1}^{\infty} S_{n}=1-M_{1} \leq 1 .
\end{aligned}
$$

And so $f \in \operatorname{Clco} \overline{\mathcal{H}} R_{q}^{m}(\lambda, \alpha, k, \gamma)$.

Conversely assume that $f \in \operatorname{Clco} \overline{\mathcal{H}} R_{q}^{m}(\lambda, \alpha, k, \gamma)$ Set.

$M_{n}=\frac{\left[(1+k)[n-1]_{q}+2+k-\alpha\right] a_{n}}{\left[(4+2 k-\alpha)+(1+k)\left|b_{1}\right|\right]|z|}[n]_{q} \varnothing(n, m)$,

$0 \leq M_{n} \leq 1, n \geq 2$.

$S_{n}=\frac{\left[(1+k)[n-1]_{q}+k+\alpha\right] b_{n}}{\left[(4+2 k-\alpha)+(1+k)\left|b_{1}\right|\right]|z|}[n]_{q} \varnothing(n, m)$,

$0 \leq S_{n} \leq 1, n \geq 1$.

Where $\sum_{n=2}^{\infty} M_{n}+\sum_{n=1}^{\infty} S_{n}=1$, so 
$M_{1}=1-\sum_{n=1}^{\infty} M_{n}+\sum_{n=1}^{\infty} S_{n}$. Therefore, $f(z)$ can be written as

$f(z)=2-\sum_{n=2}^{\infty} a_{n} z^{n}+\sum_{n=1}^{\infty} \overline{b_{n}} \overline{z^{n}}$

$=2-\sum_{n=2}^{\infty} \frac{\left[(4+2 k-\alpha)+(1+k)\left|b_{1}\right|\right]}{\left[(1+k)[n-1]_{q}+2+k-\alpha\right] \emptyset(n, m)} M_{n} z^{n}$

$+\sum_{n=1}^{\infty} \frac{\left[(4+2 k-\alpha)+(1+k)\left|b_{1}\right|\right]}{\left[(1+k)[n-1]_{q}+2+k-\alpha\right] \varnothing(n, m)} S_{n} \overline{z^{n}}$

$=z+\sum_{n=2}^{\infty}\left(h_{n}(z)-z\right) M_{n}+\sum_{n=1}^{\infty}\left(g_{n}(z)-z\right) S_{n}$

$=z\left[1-\sum_{n=2}^{\infty} M_{n}+\sum_{n=1}^{\infty} S_{n}\right]+\sum_{n=2}^{\infty}\left(h_{n}(z)-z\right) M_{n}+\sum_{n=1}^{\infty}\left(g_{n}(z)-z\right) S_{n}$

$=\sum_{n=1}^{\infty}\left(M_{n} h_{n}(z)+S_{n} g_{n}(z)\right)$.

Theorem 2.4. Let $f(z) \in \overline{\mathcal{H}} R_{q}^{m}(\lambda, \alpha, k, \gamma)$ then :

$\left(1+b_{1}\right) r+\frac{[(4+2 k-\alpha)+(1+\alpha)] b_{1}}{[2]_{q} \phi(2, m)[3+2 k-\alpha)} r^{2} \leq|f(z)| \leq\left(1-b_{1}\right) r+\frac{[(4+2 k-\alpha)+(1+\alpha)] b_{1}}{[2]_{q} \emptyset(2, m)[3+2 k-\alpha)} r^{2}$.

\section{Proof.}

We shall prove only one side, let us take the right side inequality because the proof for the left side inequality is similar way.

At first take the absolute value of the function $f(z)$, we have

$|f(z)|=\frac{[(4+2 k-\alpha)+(1+k)] b_{1}-(k-\alpha) b_{1}}{[(4+2 k-\alpha)+(1+k)] b_{1}}$

$|f(z)|=\left|z-\sum_{n=2}^{\infty} a_{n} z^{n}+\sum_{n=1}^{\infty} b_{n} \overline{z^{n}}\right|$

$\leq\left(1+b_{1}\right)|z|+\sum_{n=2}^{\infty}\left(a_{n}+b_{n}\right)|z|^{n}$

$\leq\left(1+b_{1}\right) r+\sum_{n=2}^{\infty}\left(a_{n}+b_{n}\right) r^{2}$

$\leq\left(1+b_{1}\right) r+\frac{\left[(4+2 k-\alpha)+(1+k)\left|b_{1}\right|\right]}{\emptyset(2, m)[2]_{q}[3+2 k-\alpha]} \sum_{n=2}^{\infty}\left(\frac{\emptyset(2, m)[2]_{q}[3+2 k-\alpha]}{\left[(4+2 k-\alpha)+(1+k)\left|b_{1}\right|\right]} a_{n}\right.$

$$
\left.+\frac{\emptyset(2, m)[2]_{q}[3+2 k-\alpha]}{\left[(4+2 k-\alpha)+(1+k)\left|b_{1}\right|\right]}\right) r^{2} \text {. }
$$

Then $|f(z)|=\frac{[(4+2 k-\alpha)+(1+k)] b_{1}-(k-\alpha) b_{1}}{[(4+2 k-\alpha)+(1+k)] b_{1}}$.

Theorem 2.5. The family $\overline{\mathcal{H}} R_{q}^{m}(\mathrm{~m}, \alpha, \mathrm{k}, \gamma)$ is closed for the convex combinations.

Proof. For $i=1,2,3, \ldots .$. let $f_{i}(z) \in \overline{\mathcal{H}} R_{q}^{m}(\mathrm{~m}, \alpha, \mathrm{k}, \gamma)$,

where $f_{i}(z)=z-\sum_{n=2}^{\infty} \bar{a}_{l}, n z^{n}-\sum_{n=1}^{\infty} \bar{b}_{l}, n \overline{z^{n}}$

Then by theorem : $\frac{\mathrm{A}(\mathrm{z})}{\mathrm{B}(\mathrm{z})} \geq \alpha$. 


$$
\begin{aligned}
\sum_{n=1}^{\infty}[n]_{q} \varnothing(n, m) & \frac{\left[(1+k)[n-1]_{q}+2+k+\alpha\right]}{\left[(4+2 k-\alpha)+(1+k)\left|b_{1}\right|\right]|z|} a_{i}, n \\
& +\sum_{n=1}^{\infty}[n]_{q} \emptyset(n, m)\left[(1+k)[n-1]_{q}+2+k+\alpha\right] b_{i}, n \leq 1 .
\end{aligned}
$$

For $\sum_{n=2}^{\infty} t_{i}=1,0 \leq t_{i}<1$, the convex combinations of functions $f_{i}$ may be written as$$
\sum_{i=1}^{\infty} t_{i} f_{i}(z)=z-\sum_{n=2}^{\infty}\left(\sum_{i=1}^{\infty} t_{i} a_{i}, n\right) z^{n}+\sum_{n=1}^{\infty}\left(\sum_{i=1}^{\infty} t_{i} b_{i}, n\right) \overline{z^{n}} .
$$

Using the inequality (2.4) we have :

$$
\begin{aligned}
& \sum_{n=1}^{\infty}[n]_{q} \varnothing(n, m) \frac{\left[(1+k)[n-1]_{q}+2+k+\alpha\right]}{\left[(4+2 k-\alpha)+(1+k)\left|b_{1}\right|\right]|z|}\left(\sum_{i=1}^{\infty} t_{i} a_{i}, n\right) \\
& +\sum_{n=1}^{\infty}[n]_{q} \emptyset(n, m) \frac{\left[(1+k)[n-1]_{q}+k+\alpha\right]}{\left[(4+2 k-\alpha)+(1+k)\left|b_{1}\right|\right]|z|}\left(\sum_{i=1}^{\infty} t_{i} b_{i}, n\right) \\
& =\sum_{i=1}^{\infty} t_{i}\left(\sum_{n=2}^{\infty}[n]_{q} \varnothing(n, m) \frac{\left[(1+k)[n-1]_{q}+2+k+\alpha\right]}{\left[(4+2 k-\alpha)+(1+k)\left|b_{1}\right|\right]|z|} a_{i}, n\right. \\
& \left.+\sum_{n=1}^{\infty}[n]_{q} \varnothing(n, m) \frac{\left[(1+k)[n-1]_{q}+k+\alpha\right]}{\left[(4+2 k-\alpha)+(1+k)\left|b_{1}\right|\right]|z|} b_{i}, n\right) \\
& \leq \sum_{i=1}^{\infty} t_{i}=1
\end{aligned}
$$




\section{REFERENCES}

[1] Clunie, J., \& Sheil-Small (1984), Harmonic univalent functions, Ann. Acad. Sci. Fenn. Ser. A.I. Math. 9,3, To;25.

[2] Duren, P. L.; (2001). Univalent functions (Vol. 259). Springer Science \& Business Media.

[3] Jackson, F. H; (1909). XI.- on q-functions and a certain difference operator. Earth and Environmental Science Transactions of the Royal Society of Edinburgh, 46(2), 253-281.

[4] Juma, A.S.\& Kulkarni S.R.; (2007). Harmonic Univalent Function Defined by Ruscheweyh Derivatives, International Mathematical Forum, No. 2(58),28592867.

[5] Kanas, S., \& Răducanu, D. (2014). Some class of analytic functions related to conic domains. Mathematica slovaca, 64(5), 1183-1196.

[6] Murugusundaramoorthy, G., \& Jahangiri, J. M. (2019). Ruscheweyh-Type Harmonic Functions Defined By q-Differential Operators. Khayyam Journal of Mathematics, 5(1), 79-88.

[7] Ruscheweyh, S; (1975). New criteria for univalent functions. Proceedings of the American Mathematical Society, 49(1),109-115. 